\title{
Editorial
}

\section{Systems biology: a way to make complex problems more understandable}

\author{
IN Lavrik ${ }^{1,2}$ and B Zhivotovsky ${ }^{\star, 2,3}$ \\ Cell Death and Disease (2014) 5, e1256; doi:10.1038/cddis.2014.195; published online 29 May 2014
}

Despite major advances in biomedical research, exceptional difficulties remain arising from the complexity of various diseases and their variability. Development of many disorders and their therapeutic responses are associated with disturbances in cell death pathways. Although for $>40$ years our knowledge about cell death was restricted to apoptosis and pathological necrosis, nowadays based on Recommendations of the Nomenclature Committee of Cell Death, there are $>10$ different cell death modalities recognized. ${ }^{1}$ Cell death was classified according to its morphological appearance, enzymological criteria, functional aspects or immunological characteristics. ${ }^{2}$ Intensive work in the field, for example, by the beginning of $2014>400000$ publications in PubMed are related to this area of research, led to understanding the biochemical features of various modes of cell death and some of molecular mechanisms of their activation/development/ execution.

To get the insight into the complexity of the cell death networks, the upcoming field of systems biology has been successfully employed over the past decade. Systems biology is an interdisciplinary field of research that focuses on complex interactions within biological structures using a holistic perspective approach to biological and biomedical investigations. Systems biology combines theoretical and computational approaches with quantitative experimental data. On the theoretical side, a wide spectrum of mathematical formalisms is used. Their choice is based on the question to be answered by the modeling, available experimental data sets and the intricacy of the signaling network under consideration. Boolean models are effectively used to characterize large cell death signaling networks. In Boolean modeling, protein activities are presented by nodes that can be either off or on, and no knowledge is required for the quantitative characteristics of the individual reactions. In contrast, ordinary differential equations (ODEs) describe temporal dynamics of signaling networks and require the knowledge of kinetic parameters of the system as well as a set of temporally solved experimental data. ODE-based modeling is one of the most common approaches used in the analysis of the cell death networks. ODEs might not be sufficient for modeling spatiotemporal processes within the cell, for example, translocations within different compartments that involve spatiotemporal gradients. ${ }^{3}$ In this case modeling is conducted using partial differential equations (PDEs). Modeling cell-to-cell variations arising from single-cell measurements requires stochastic simulations. In addition, Petri nets, agent-based models (ABMs) and Bayesian models have been employed for the analysis of the cell death networks. The combination of various mathematical tools allowed to quantitatively describe the major cell death processes and to identify biologically relevant systems' properties that will be highlighted in this issue.

Computational models require the exact knowledge about the numbers and interaction constants of the molecules in the pathway that allows making unique quantitative assessments upon molecular mechanisms of the complex signaling network regulation. These vigorous quantifications require state-of-the-art experimental methodology that includes quantitative biochemistry, cell biology and mass spectrometry techniques. The classical western blot and immunoprecipitation approaches were recently developed in the systems biology studies to generate time-resolved population data on the semiquantitative and quantitative levels. Single-cell analysis has enabled valuable insights into cell death using a number of special tools, including FRET-based and localization-based caspase activity probes. Finally, progress in mass spectrometry field is coming up with AQUA- and SILAC-based technologies, and development toward singlecell mass spectrometry analysis provides yet another major technological advance essential for the quantitative data generation.

During the past decade, the powerful methodology of systems biology combining high-level mathematics with the state-of-the-art quantitative experimental work helped us to understand many aspects of cell's decision to live or to die, in particular, death receptor- and mitochondria-mediated cell death pathways were elucidated and understood on a systems level with the unprecedented level of detail. ${ }^{4-6}$

Extrinsic apoptosis is triggered by activation of the death receptors. Modeling death receptor network provided the first example of systems biology study of cell death supported by experimental data. ${ }^{7}$ This pioneer study was followed by a

\footnotetext{
${ }^{1}$ Department of Translational Inflammation, Institute of Experimental Internal Medicine, Otto von Guericke University, Magdeburg, Germany; ${ }^{2}$ Faculty of Basic Medicine, MV Lomonosov Moscow State University, Moscow, Russia and ${ }^{3}$ Division of Toxicology, Institute of Environmental Medicine, Karolinska Institutet, Box 210, Stockholm, Sweden

${ }^{*}$ Corresponding author: Dr B Zhivotovsky, Division of Toxicology, Institute of Environmental Medicine, Karolinska Institutet, Box 210, SE 171 77 Stockholm, Sweden. Tel: +46 8524 87588; Fax: +46 8329 041; E-mail: Boris.Zhivotovsky@ki.se
} 
number of models uncovering dynamics of death receptor signaling and death-inducing signaling complex (DISC), the latter is essential for the initiation of CD95-mediated apoptotic and non-apoptotic responses. ${ }^{8-10}$ Furthermore, recently, the stoichiometry of CD95 and TRAIL-R DISC complexes was revealed by quantitative and systems biology approaches. ${ }^{4,5}$ Thus, there is a significant progress in this field, which is essential in the context of normal cell physiology and disease.

The term 'apoptosome' was introduced in 1997 by Hengartner ${ }^{11}$ and used to describe the second molecular complex, which is activated during mitochondria-mediated apoptosis. In the presence of dATP, cytochrome $c$, released from the mitochondria, interacts with Apaf-1 and procaspase-9 leading to activation of this high-molecular weight complex. Release of cytochrome $c$ is regulated by various mechanisms, which involve function of several pro- and anti-apoptotic members from $\mathrm{Bcl}-2$ family proteins. Interplay within these proteins is complex and mathematical modeling of this complexity was able to dissect spatiotemporal activation of apoptosomedependent pathway. ${ }^{3}$ Importantly, there exists a direct connection between functions of DISC and apoptosome complexes, which requires $\mathrm{BH} 3$-only proteins of $\mathrm{Bcl}-2$ family. ${ }^{12}$

Accumulating evidence revealed the crosstalk not only within apoptosis, but also between various cell death modalities. Natural (mutations) or artificial (chemical inhibitors) inactivation of caspases might cause the shift from apoptosis to necroptosis, or to the mixture of these two cell death modes. Cleavage of Atg5 (essential for development of autophagy) causes shift from autophagy to apoptosis. ${ }^{13}$ Depending on the type of lethal stimulus, the cell death process can be initiated in different intracellular compartments, and their crosstalk is essential for all cell death modalities. The inter-organelle crosstalk involves several molecular switches within the signaling networks. ${ }^{14,15}$ Among proteins regulating this type of switches is p53, which, depending on localization, can be involved in regulation of apoptosis, autophagy or necroptosis. Interestingly, Bcl-2 family proteins are essential for the regulation of a majority of programmed cell death modalities. The complexity of balance between different cell death modalities is supported by the observation that nature and severity of stimulus might influence the inter-organelle crosstalk. In some cases suppression of the function of a particular intracellular compartment might switch one mode of cell death to another. For example, inhibition of mitochondrial energy metabolism can shift from apoptosis to necrosis. It seems that the point of no return in many cell death modalities is similar and associated with mitochondria. Systems biology can be applied not only to learn more about the molecular mechanisms regulating various cell death modalities, but also their crosstalk.

As mentioned above, disturbances in cell death pathways result in pathogenesis of various disorders. Thus, too much cell death is associated with neurological (Parkinson's, Alzheimer's and Huntington's) diseases and immunological disorders (AIDS). On the other hand, too little cell death is associated with development of malignancies. Importantly, there are many pathological situations when crosstalk between different cell death subroutines has a significant role, and treatment of all of these disorders is based on the attempt to either suppress or induce cell death. Thus, suppression of autophagy is followed by the accumulation of
ROS, which makes tumor cells more sensitive to apoptosis. ${ }^{16}$ Pharmacological inhibition of autophagy does not prevent Atg5-dependent mitotic catastrophe, but shifts the balance to caspase-dependent death of tumor cells. ${ }^{17}$ To understand the mechanism of complex crosstalks, especially, in multifactorial disorders, an integrated systems biology approach can be applied. In the following several review articles, published under the title of 'Systems Biology approach to investigate cell death pathways in diseases' different angles of systems biology requirement for cell death research and medical applications are discussed. Thus, Lavrik ${ }^{18}$ summarizes knowledge of how systems biology of death receptor networks is operating in making decision between life and death. On the other hand, Rehm et al. ${ }^{19}$ discuss achievement, perspectives and challenges in systems medicine that can arrive from computational modeling of the intrinsic apoptosis pathway essential for analysis of chemotherapy resistance.

Natural and acquired resistance of tumor cells to well-known therapies in many cases is also associated with dysregulation of apoptosis. The mechanisms of this resistance are different and complex. Cisplatin is often employed for the clinical treatment of patients with various tumors; however, in spite of high rates of clinical responses in many cases tumor cells activate an adaptive response to cisplatin that makes them less susceptible to the antiproliferative and cytotoxic effects to this drug. Recently, many systems biology studies took attempts to resolve the complex problem of cisplatin resistance. These studies are summarized to a great level of detail by Galuzzi et al. $^{20}$ who are trying to understand how obtained knowledge can be used in the development of rational approaches to tackle the clinically relevant problem of cisplatin resistance.

Although major advances have been made in the diagnosis of different tumor types, it is still important to understand the precise functioning of signaling pathways associated with resistance to current treatments and develop biomarkers essential for better diagnostics and prediction of tumor response. Using lung cancer as an example, Viktorsson et al. $^{21}$ are trying to explain how systems biology-based approaches can help in generation of new biomarkers and novel therapeutic targets in this devastating disorder, or even contribution to personalized treatment of this malignancy.

Addressing the question of responsiveness of tumor cells to therapy, one should keep in mind the problem of not simultaneous/uniform response. Many factors can explain this diversity of cellular reactions. Analysis of systems biology models enables to take a general view on the cell death process to identify multifactorial determinants of the cell death decision. Xia et al. ${ }^{22}$ discuss how 'systems pharmacology' could help in the development of combinatory treatment strategies to either manipulate the state of cancer cells in order to sensitize them to apoptosis-inducing drugs or manipulate the dynamics of signaling that encode specific cellular responses, compelling cancer cells toward apoptosis.

It is known that the main goal of systems biology is to find the most rational route; therefore, a unified 'language' (model) should be used by bioinformaticians to include all available information essential for understanding the proper functioning of the biological system. One of the aims for researchers in cell death field is to build and validate models to define proper functioning of cell death machinery and systemic 
understanding of cell death pathways to refine the molecular diagnosis of various types of disease associated with dysfunction of cell death. It will help in optimizing the calculation of prognostic and predictive parameters of guiding new strategies to ameliorate existing treatments and identification of novel targets for therapeutic modulation of cell death pathways. We hope that some questions related to that are addressed in the following reviews.

\section{Conflict of Interest}

The authors declare no conflict of interest.

Acknowledgements. The work in the authors group was supported by grants from the Swedish and the Stockholm Cancer Societies, the Swedish Childhood Cancer Foundation, the Swedish Research Council and Russian Research Foundation (to BZ) and the Ministry of Sciences and Economic Affairs of Saxony-Anhalt (MW-21LMS 5), BMBF (eBIO project "ImmunoQuant" - TPU - 0316170G), the Helmholtz-Russia Joint Research Groups - 2008-2 and RFFI 14-04-00699 (to INL).

1. Kroemer $\mathrm{G}$ et al. Cell Death Differ 2009; 16: 3-11.

2. Galluzzi L et al. Cell Death Differ 2012; 19: 107-120.

3. Rehm M et al. Cell Death Differ 2009; 16: 613-623.

4. Dickens LS et al. Mol Cell 2012; 47: 291-305.

5. Schleich K et al. Mol Cell 2012; 47: 306-319.

6. Wurstle ML, Laussmann MA, Rehm M. Exp Cell Res 2012; 318: 1213-1220.
7. Bentele M et al. J Cell Biol 2004; 166: 839-851.

8. Muzio M et al. Cell 1996; 85: 817-827.

9. Boldin MP et al. Cell 1996; 85: 803-815.

10. Walczak H et al. Nat Med 1999; 5: 157-163.

11. Hengartner MO. Nature 1997; 388: 714-715.

12. Li H et al. Cell 1998; 94: 491-501.

13. Yousefi S et al. Nat Cell Biol 2006; 8: 1124-1132.

14. Calzone L et al. PLoS Comput Biol 2010; 6: e1000702.

15. Rubinstein AD, Kimchi A. J Cell Sci 2012; 125: 5259-5268.

16. Kaminskyy VO et al. Autophagy 2012; 8: 1032-1044.

17. Maskey D et al. Nat Commun 2013; 4: 2130.

18. Lavrik IN. Cell Death Dis 2014; 5: e1208.

19. Würstle ML et al. Cell Death Dis 2014; doi:10.1038/cddis.2014.36.

20. Galluzzi L et al. Cell Death Dis 2014; doi:10.1038/cddis.2013.428.

21. Viktorsson K et al. Cell Death Dis 2014; doi:10.1038/cddis.2014.28.

22. Xia X et al. Cell Death Dis 2014; doi:10.1038/cddis.2014.199.

(c) (i) $(\theta)$ Cell Death and Disease is an open-access journal published by Nature Publishing Group. This work is licensed under a Creative Commons Attribution-NonCommercialNoDerivs 3.0 Unported License. The images or other third party material in this article are included in the article's Creative Commons license, unless indicated otherwise in the credit line; if the material is not included under the Creative Commons license, users will need to obtain permission from the license holder to reproduce the material. To view a copy of this license, visit http://creativecommons.org/licenses/ by-nc-nd/3.0/ 